\title{
Symptomatic Medial Exostosis of the Great Toe Distal Phalanx: A Complication Due to Over-correction Following Akin Osteotomy for Hallux Valgus Repair
}

Carlos Villas, MD, PhD, ${ }^{1}$ Javier Del Río, MD, ${ }^{3}$ Andres Valenti, MD, ${ }^{3}$ and Matías Alfonso, $\mathrm{MD}, \mathrm{PhD}^{2}$

${ }^{1}$ Consultant and Professor, Orthopaedic Surgery, Clínica Universitaria de Navarra, Pamplona, Navarra, Spain.

2 Consultant, Orthopaedic Surgery, Clínica Universitaria de Navarra, Navarra, Pamplana, Spain.

${ }^{3}$ Resident, Orthopaedic Surgery, Clínica Universtaria de Navarra, Navarra, Pamplona, Spain.

Address correspondence to: Carlos Villas, Consultant and Professor, Clínica Universitaria de Navarra, Orthopedic Surgery Department, Pio XII sn, 31008 Pamplona, Navarra, Spain. E-mail: cvillas@unav.es

\begin{abstract}
The authors present the case of a 54-year-old female who developed a painful compression lesion localized to the medial aspect of the base of the distal phalanx of the great toe as a complication of hallux valgus surgery. Preoperative radiographic evaluation of the patient's foot revealed the first ray to be longer than the second, a $12^{\circ}$ first intermetatarsal angle, a $33^{\circ}$ hallux abductus angle, and an exostosis at the medial aspect of the base of the hallux that was not considered by the surgeon to be important. Correction of the hallux valgus deformity was performed with a combination of scarf and Akin osteotomies, and the intermetatarsal and hallux abductus angles reduced to $2^{\circ}$ and $8^{\circ}$, respectively. By 2 months postoperative, the patient was complaining of pain at the medial aspect of the distal phalanx of the hallux associated with shoe pressure. The pain correlated both clinically and radiologically with the exostosis at the base of the distal phalanx, and had become symptomatic only after the hallux had been operatively realigned. At 6 months postoperative, percutaneous exostectomy was undertaken to remove the exostosis. Pain relief was complete, thereafter, and after 2 years of postoperative follow-up the patient remained pain free. The clinical importance of a medial exostosis localized to the base of the distal phalanx of the hallux must be taken into consideration whenever hallux valgus correction is undertaken, and this is particularly important whenever an Akin osteotomy is being considered. Level of Clinical Evidence: 4
\end{abstract}

\section{KEY WORDS}

Akin osteotomy, exostosis, hallux valgus, phalangeal horn, postoperative complication 


\section{INTRODUCTION}

A variety of surgical procedures can be used to treat symptomatic hallux abductovalgus (HAV, hallux valgus), and the use of metatarsal and phalangeal osteotomies has long been commonplace in this realm. As a corollary to the widespread use of osteotomies to correct hallux valgus, a wide array of potential postoperative complications are known to exist (1-4). In some cases, it is possible to identify in the preoperative period conditions that could potentially cause a postoperative problem, and it is the surgeon's responsibility to consider those items that could reasonably result in such a complication. In the case described in this report, the presence of a medial exostosis localized to the base of the distal phalanx of the hallux was not considered to be a potential complicating factor in a patient suffering with hallux valgus deformity. However, in the postoperative phase, this preexisting bony prominence became painful in response to shoe pressure, and ultimately the decision was made to return to the operating room to excise the exostosis. It is the purpose of this report to make clear the potential problem associated with the presence of a medial exostosis localized to the base of the distal hallucial phalanx in patients undergoing surgical repair of the deformity of hallux abductovalgus.

\section{CASE REPORT}

A 54-year-old female, who worked as a nurse at our hospital, presented for surgical correction of her right hallux valgus deformity. She complained of pain localized to the dorsal and medial aspects of her first metatarsal head, which persisted even after conservative therapeutic measures, including the use of wider and softer shoes, padding, and oral anti-inflammatory and analgesic medications, had failed to provide sufficient pain relief. The physical examination revealed bilateral hallux valgus deformities with very long great toes, consistent with the Egyptian digital morphotype (5-7), and she complained of pain upon palpation of the bunion at the first metatarsal of the right foot.

Plain film radiographs of the right foot displayed the long first toe relative to the second, a $12^{\circ}$ intermetatarsal angle, a $33^{\circ}$ hallux valgus angle, and an exostosis at the medial margin of the base of the distal phalanx of the great toe (Figure 1a, b, and c). After discussion of the surgical options and potential risks and complications, surgical correction of the hallux valgus deformity was performed by means of a scarf osteotomy $(8,9)$ of the first metatarsal combined with the use of an Akin adductory closing-wedge osteotomy (10) of the proximal phalanx. The surgery proceeded without apparent incident, and so too did the initial postoperative healing phase. Following the operation, the hallux remained loner than the second toe, and the first intermetatarsal and hallux abductus angles were reduced to $8^{\circ}$ and $2^{\circ}$, respectively; and the alignment of the base of the proximal phalanx of the hallux on the first metatarsal head was considered to be satisfactory (Figure 2, A).

However, as time progressed in the postoperative phase, the patient began to complain of pain localized to the medial aspect of the hallux, and this was aggravated by shoe contact and direct pressure. The pain was that of a deep aching sensation, without paresthesia or radiation proximally or distally, and there was no clinical evidence of involvement of either the plantar or dorsal proper digital cutaneous nerves due to compression. By 8 weeks following the surgery, she displayed continued to display 
mild edema in association with the operative site, and she was encouraged to try and avoid shoe pressure at the sensitive site, and to see if the use of oral ibuprofen (600 $\mathrm{mg}$ by mouth up to 3 times daily with food) would help. Nonetheless, her pain persisted and it became clinically and radiographically obvious that it was associated with the persistent exostosis at the medial aspect of the base of the distal hallucial phalanx (Figure 2, B).

At approximately 6 months following the surgery for repair of her hallux valgus deformity, an additional operation was undertaken to remove the exostosis of the phalangeal base by means of percutaneous exostectomy performed using local anesthesia combined with intravenous sedation. The lesion was excised using a sharp bone nipper and a rasp, after which the wound was closed in layers and the patient allowed to ambulate full weight bearing in a bandage and postoperative shoe. Postoperative radiographs revealed complete removal of the prominent portion of the medial aspect of the base of the distal phalanx (Figure 2, C), and the patient healed unremarkable thereafter and resumed all of her regular activities and the use of any shoes that she desired to wear.

\section{DISCUSSION}

A variety of complications of hallux valgus surgery have been described, ranging from general complications such as wound infection and venous thrombosis (11), to complications that are more exclusively related to bunion repair, such as recurrence of the deformity, overcorrection, transfer metatarsalgia, lesser metatarsal stress fracture (12), hallux varus, joint stiffness, and malunion or nonunion at the sites of osteotomies used to correct the deformity $(1,13)$. In fact, it has been reported that perioperative complications occur in approximately $6 \%$ of patients undergoing scarf osteotomy (14). In the patient described in this case report, it is our opinion that her postoperative pain, which was new to the patient and had not been present prior to the surgery to correct her bunion, was iatrogenic in that the preexisting, prominent medial aspect of the base of the distal hallucial phalanx only become clinically prominent after the hallux was adducted relative to the long axis of the first metatarsal. More specifically, we feel that over-correction of the hallux interphalangeus angle led to a clinically relevant prominence of the exostosis that, after non-surgical treatment, was only resolved satisfactorily with a second operation directed at removal of the bony prominence. Although our patient underwent an Akin proximal phalangeal osteotomy combined with a scarf first metatarsal osteotomy, we do not feel that the scarf procedure alone would have led to a symptomatic prominence of the distal phalangeal medial exostosis in this case (although it could be possible if the exostosis were large enough and the relative position of the proximal phalanx on the metatarsal head were adducted enough from the preoperative hallux abductus alignment).

To the best of our knowledge, symptomatic overcorrection of the great toe due to the presence of a preexisting distal phalangeal medial exostosis, in association with hallux valgus repair has not been described in the published literature prior to this case report. Although the complication was rectified by means of returning to the operating room to resect the exostosis, it is likely that this problem could have been avoided had the importance of the medial exostosis been recognized prior to the first surgical intervention. Moreover, we did not put much emphasis on the hallux abductus 
interphalangeus angle in the period preceding the first operation, and we realize that it could be argued that the Akin osteotomy, while certainly being likely to make the hallux look straighter in the postoperative period, due to adduction of the distal portion of the proximal phalanx, may not have been necessary in this case due to the preoperative structural alignment of the proximal phalanx. It is our experience, however, that patients are generally well satisfied when the hallux clinically appears to be straight relative to the second toe, and that the goal of achieving this straightened appearance of the great toe often outweighs the need to thoroughly balance the relationship of the base of the proximal phalanx with the metatarsal head, as long as the first metatarsophalangeal joint and the first intermetatarsal angle, hence the prominence of the first metatarsal head, are reasonably well aligned. We also understand that the desire to achieve a hallux that clinically appears to be straight may, as it appears to have done in the case described in this report, predispose to overuse of the Akin osteotomy. Furthermore, we feel that we underestimated the impact that the exostosis located at the medial aspect of the base of the distal phalanx would have on our patient, after we straightened the hallux. For this reason, we encourage surgeons to be wary of this potential complication whenever surgical efforts focus on straightening the hallux. If an exostosis is present (Figure 3a, b), or even in the presence of a hypertrophic medial condyle at the base of the distal phalanx or at the head of the proximal phalanx, we feel that consideration should be given to surgical excision, in a prophylactic sense, of the potentially prominent bone whenever hallux valgus correction is undertaken. Still further, we feel that this potential outcome should be discussed with the patient prior to operative repair of hallux valgus, so that they understand that additional surgery may be indicated at the time that the bunionectomy is performed, or at a later date should the exostosis ever become painful.

Financial Disclosure: None reported.

Conflict of Interest: None reported.

\section{REFERENCES}

1. Kilmartin TE. Revision of failed foot surgery: a critical analysis. J Foot Ankle Surg 41:309-311, 2002.

2. Kitaoka HB. Arthrodesis versus resection arthroplasty for failed hallux valgus operations. Clin Orthop Relat Res 347:208-214, 1998.

3. Machacek F Jr. Salvage of a failed Keller resection arthroplasty. J Bone Joint Surg Am 86A:1131-1138, 2004.

4. Trnka HJ. Arthrodesis procedures for salvage of the hallux metatarsophalangeal joint. Foot Ankle Clin 5:673-686, 2000.

5. Jarde O, Trinquier JL, Mertl P, Filloux JF, Vives P. [Hallux valgus treated by shortening of the first phalanx with trans-epiphyseal impaction and adductor plasty. Apropos of 49 cases with 5-years follow-up]. Rev Chir Orthop Reparatrice Appar Mot 81(2):136-141, 1995. French.

6. Regnauld B. Patologia elettiva dei metatarsi (Pathology of elective metatarsals). In Chirurgia del Piede (Foot Surgery), pp 277-279, edited by A Gaggi, Bologna, 1997. 
7. Kelikian H. Hallux Valgus, Allied Deformities of the Forefoot and Metatarsalgia, p 339, WB Saunders Co, Philadelphia, 1965.

8. Barouk LS. Scarf and Great Toe Osteotomies in the Treatment of Hallux Valgus: Forefoot Reconstruction, pp 92-97, Springer, Paris, 2003.

9. Jones S. Scarf osteotomy for hallux valgus. J Bone Joint Surg Br 86B:830836, 2004.

10. Frey C. The Akin procedure: an analysis of results. Foot Ankle 12:1-6, 1991.

11. Radl R. Venous thrombosis after hallux valgus surgery. J Bone Joint Surg 85A:1204-1208, 2003.

12. Danon G. An unusual complication of the Keller bunionectomy: spontaneous stress fractures of all lesser metatarsals. J Foot Surg 28:335-339, 1989.

13. Sammarco G. Complications after surgery of the hallux. Clin Orthop Relat Res 391:59-71, 2001.

14. Smith AM, Alwan T, Davies MS. Perioperative complications of the Scarf osteotomy. Foot Ankle Int 24:222-227, 2003. 


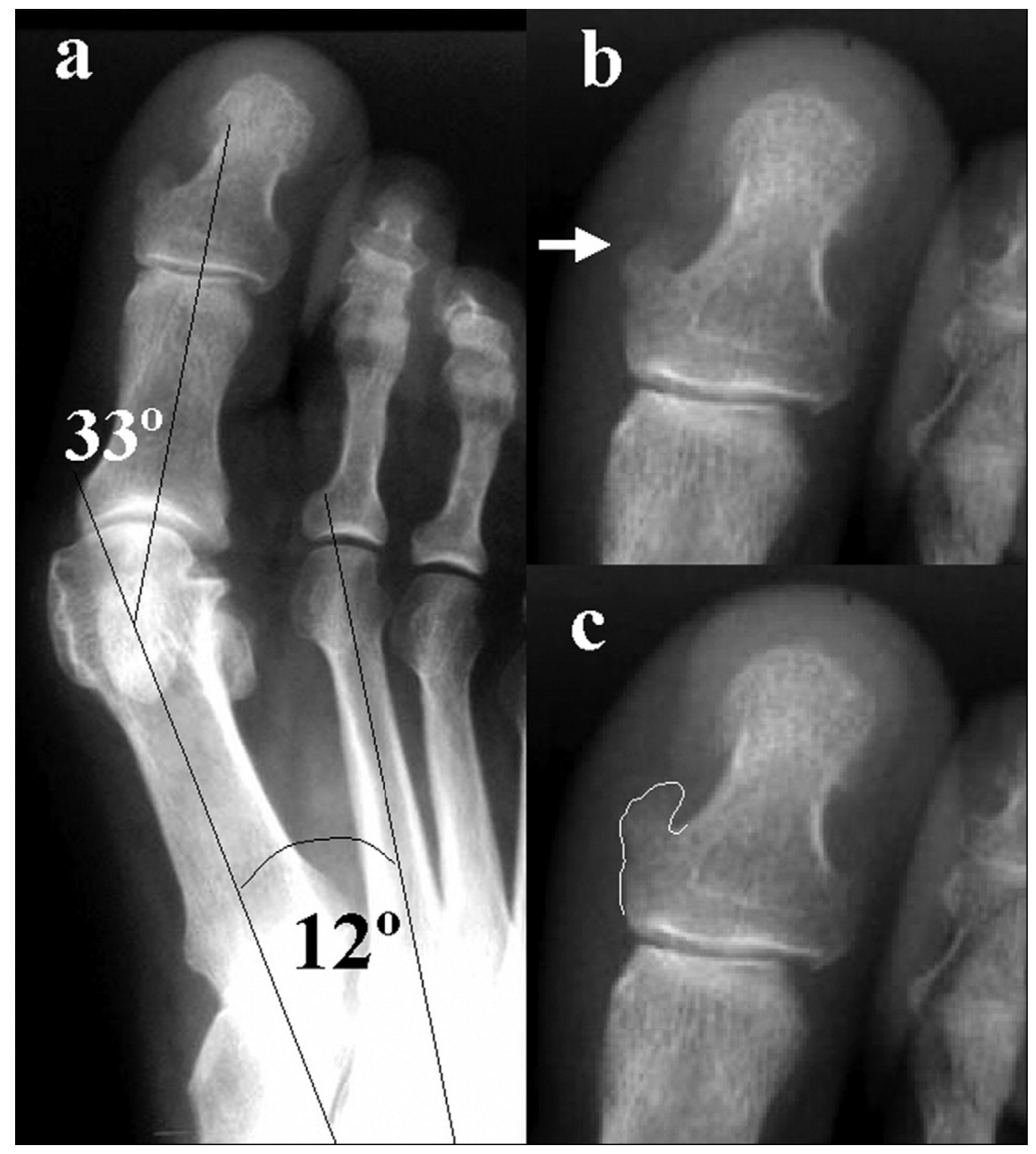

Figure 1. Preoperative view showing (a) the valgus deformity and its quantification, (b) a detail of the exostosis (arrow), and (c) the same image outlined. 


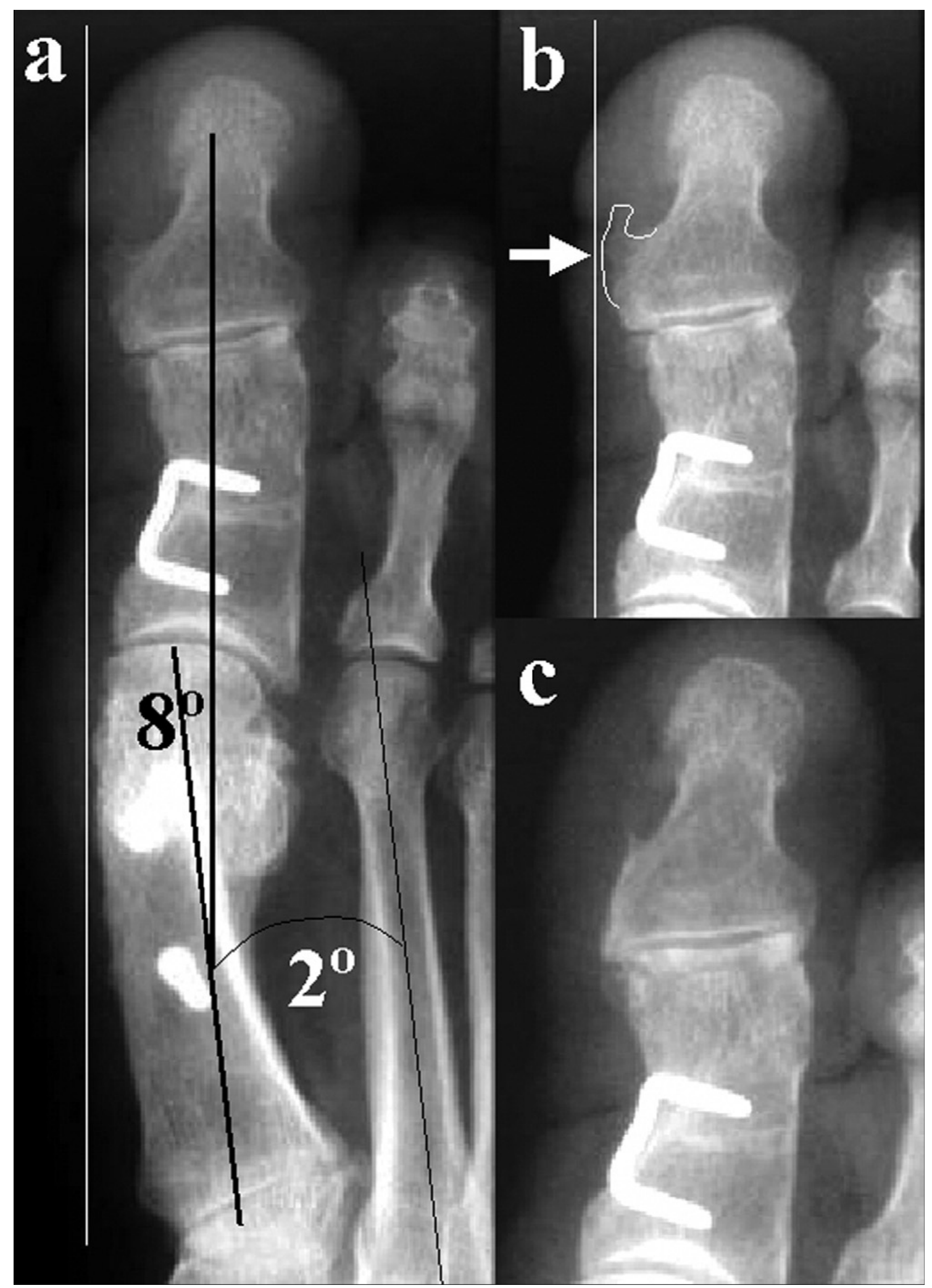

Figure 2. Postoperative view showing (a) overcorrection at the first phalanx, (b) a detail of the theoretical foot-shoe contact line outlining the realignment at the level of the exostosis, and (c) detailed view after resection of the exostosis. 


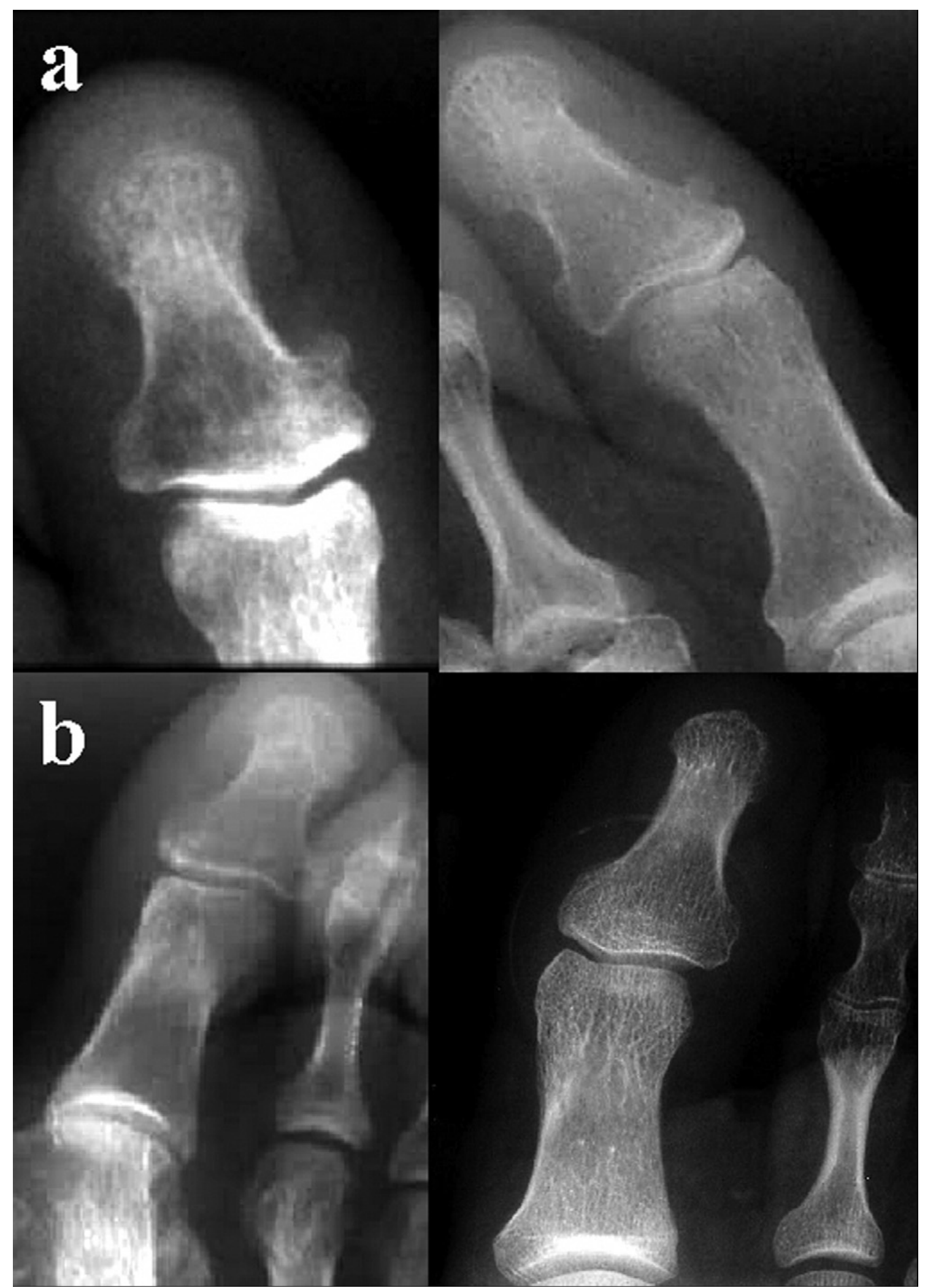

Figure 3. Images of distal phalanges of great toes belonging to different asymptomatic patients with (a), and without (b), a prominent exostosis localized to the medial aspect of the base of the distal hallucial phalanx. 